\title{
Optimal Training Design for Channel Estimation in OFDM/OQAM Cooperative Systems
}

\author{
Christos Mavrokefalidis ${ }^{*}$, Eleftherios Kofidis ${ }^{\dagger *}$, Athanasios Rontogiannis ${ }^{\ddagger *}$, and Sergios Theodoridis ${ }^{\S *}$ \\ *Computer Technology Institute and Press "Diophantus", \\ Patras University Campus, Patras, Greece (corr. author; maurokef@ceid.upatras.gr) \\ ${ }^{\dagger}$ Dept. of Statistics and Insurance Science, \\ University of Piraeus, Piraeus, Greece (kofidis@unipi.gr) \\ ${ }^{\ddagger}$ Institute for Astronomy, Astrophysics, Space Applications and Remote Sensing, \\ National Observatory of Athens, Athens, Greece (tronto@noa.gr) \\ $\S$ Dept. of Informatics and Telecommunications, \\ University of Athens, Athens, Greece (stheodor@di.uoa.gr)
}

\begin{abstract}
Channel estimation in OFDM/OQAM-based cooperative systems is considered in this paper. Cooperation is based on a single AF relay, and a well established two-phase protocol is adopted for its operation. The problem of optimally designing the preambles in the two phases is investigated for LS channel estimation, where optimality is in the sense of minimum MSE subject to a transmit energy constraint. Optimal conditions are derived for the energy allocations and positions of the pilot symbols. Equalization is also considered, where a relay-induced interference term is identified at the destination node. A simple cancellation procedure is proposed and evaluated. Simulation results are reported that corroborate the analysis and provide a comparison with the corresponding CP-OFDM system.
\end{abstract}

Keywords-Channel estimation, filter bank-based multicarrier (FBMC), least squares (LS), offset quadrature amplitude modulation (OQAM), orthogonal frequency division multiplexing (OFDM), relaying networks.

\section{INTRODUCTION}

Cooperative communication systems have received increasing attention during the last decade or so, due to their ability to offer significant capacity and spatial diversity gains with relatively simple single-antenna terminals [4], [5]. Multicarrier modulation, namely orthogonal frequency division multiplexing (OFDM), is also commonly adopted in such systems to cope with the frequency selectivity of the channels involved. The popularity of OFDM mainly comes from its ability to turn a frequency selective channel into an equivalent set of parallel flat channels with independent noises, thus considerably simplifying the receiver's tasks in estimating and equalizing the channel [12]. This is achieved with the use of a guard interval, most commonly a cyclic prefix (CP), which however results in a power and spectral efficiency loss (as high as $25 \%$ ). The bad frequency localization (with their high sidelobes) of the OFDM filters is responsible for further drawbacks, including increased sensitivity to Doppler effects, spectral leakage and difficulties in user synchronization. Notably, the latter are of great importance in cooperative systems, where the synchronization requirements are desired to be minimal.

Multicarrier schemes based on filter banks (FBMC) have recently shown the potential of correcting these drawbacks, thus providing an attractive alternative to OFDM, at the

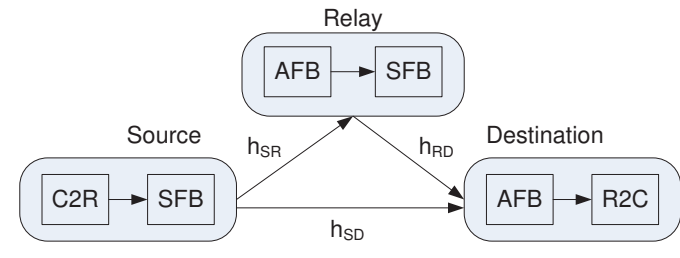

Fig. 1. The cooperative system under consideration.

cost of some additional complexity and delay [13]. FBMC offers significantly better spectral containment (via the use of carefully designed pulse shaping filters) and hence, among other gains, relaxed synchronization requirements. When combined with offset quadrature amplitude modulation (OQAM), filters with good localization in both time and frequency are possible, resulting in the so-called OFDM/OQAM modulation scheme [15]. The latter avoids the use of CP altogether and has the potential of a maximum spectral efficiency. Recently, impressive improvements in the throughput of cognitive radio relaying networks employing OFDM/OQAM were demonstrated over their CP-OFDM counterparts [14].

OFDM/OQAM suffers, however, from an imaginary intercarrier/intersymbol interference, that complicates signal processing tasks at the receiver, including channel estimation. A multitude of training designs and associated channel estimation methods have been proposed for such systems [8]. The design of optimal OFDM/OQAM preambles for the purpose of estimating the channel in single-antenna single-link channels was investigated in [6] (see also [7], [8]). Both full (i.e., with pilots at all the subcarriers) and sparse (i.e., with isolated pilot subcarriers surrounded by nulls) preambles ${ }^{1}$ were considered and their performances were analyzed.

The goal of this paper is to address the above problem for the first time in the context of a simple cooperative network as the one given in Fig. 1. Single-antenna transmitters and receivers are assumed, while a single one-way relay is employed to assist the transmission, following a simple amplify-and-forward (AF) protocol. The system operates in

\footnotetext{
${ }^{1}$ Known also in the OFDM literature as block-type and comb-type, respec-
} tively [6]. 
two phases, with the source trasmitting to the destination in both of them. In the second phase, the relay forwards to the destination an amplified version of the signal transmitted by the source in the first phase. This allows the first phase signal to be received through two different links, thus enhancing the diversity. In a manner analogous to a CP-OFDM-based system, filter banks are employed at the relay terminal to help amplify the received signal per subcarrier. It is desired to estimate the channels in both of the paths leading to the destination node. In [10], the problem of optimal sparse preamble design for least squares (LS) channel estimation in such a system based on CP-OFDM was studied, where optimality is with respect to the mean squared estimation error (MSE) with a constraint on the total transmitted energy. The same problem is addressed in this paper, for the FBMC-based system of Fig. 1, following an approach similar to the one of [10] and [6]. The resulting optimality conditions are analogous to those derived in [10] and dictate that the source should allocate the whole of the training energy in the first phase, to equispaced and equipowered pilots. Moreover, the relay should also uniformly allocate its energy to forward the corresponding training signal. These results agree with those reported in the earlier works. Nevertheless, the presence of the intrinsic interference in the OFDM/OQAM system makes it a nontrivial task to define AF at the relay and also results in a relay-induced interference term at the destination receiver, which prevents data recovery. A simple procedure to cancel this term out is proposed and shown to be effective. The reported simulation results corroborate the analysis and demonstrate a performance similar to that of the CP-OFDM system.

\section{System DESCRIPTION}

\section{A. The OFDM/OQAM System}

The (baseband) output signal of an OFDM/OQAM synthesis filter bank (SFB) is given by [15]

$$
s(l)=\sum_{m=0}^{M-1} \sum_{n} a_{m, n} g_{m, n}(l),
$$

where $a_{m, n}$ are real OQAM symbols (resulting from a complex to real (C2R) OQAM modulator, as shown in Fig. 1) and

$$
g_{m, n}(l)=g\left(l-n \frac{M}{2}\right) e^{j \frac{2 \pi}{M} m\left(l-\frac{L_{g}-1}{2}\right)} e^{j \varphi_{m, n}},
$$

with $g$ being the real symmetric prototype filter impulse response (assumed here of unit energy) of length $L_{g}, M$ being the even number of subcarriers, and $\varphi_{m, n}=\varphi_{0}+\frac{\pi}{2}(m+$ $n) \bmod \pi$, where $\varphi_{0}$ can be arbitrarily chosen [15]. The filter $g$ is usually designed to have length $L_{g}=K M$, with $K$ being the overlapping factor. The double subscript $(\cdot)_{m, n}$ denotes the $(m, n)$-th frequency-time (FT) point. Thus, $m$ is the subcarrier index and $n$ the OFDM/OQAM symbol time index.

The cost paid in such a system for its advantages over other FBMC schemes is that its subcarrier functions $g_{m, n}$ are orthogonal in the real field only. This implies that even in the absence of channel distortion and noise, and with perfect time and frequency synchronization, there will be some intercarrier (and/or intersymbol) interference at the output of the analysis filter bank (AFB), which is purely imaginary and known as intrinsic interference [8]. We will make here the common assumption that the channel is (approximately) frequency flat over each subband. Moreover, the interference from FT points outside the first-order neighborhood $\Omega(p, q)$ of the FT point $(p, q)$ (excluding $(p, q)$ itself) will be assumed to be negligible, a common assumption, valid for pulses $g$ that are well localized in both time and frequency. If, moreover, the channel frequency response (CFR) is almost constant over this neighborhood, one can write the AFB output at $(p, q)$ as [8]

$$
y(p, q) \approx H(p, q) c(p, q)+\eta(p, q),
$$

where $H(p, q)$ is the (M-point) CFR at that point,

$$
c(p, q)=a(p, q)+j \sum_{(m, n) \in \Omega_{p, q}} a(m, n)\langle g\rangle_{m, n}^{p, q}
$$

is the virtual transmitted symbol at $(p, q)$, with $\langle g\rangle_{m, n}^{p, q}$ denoting the imaginary part of the transmultiplexer transfer function, and $\eta(p, q)$ is the associated noise component. The latter has been shown to be stationary and correlated among subcarriers (also Gaussian with zero mean and variance $\sigma^{2}$ for a channel noise with these statistics) (see [3]). In the sequel, this correlation will be mostly neglected, as it is the case with well localized AFBs.

\section{B. The Cooperative System}

The cooperative system under consideration is schematically shown in Fig. 1. A two-phase transmission protocol (first proposed in [11]) as described previously is adopted. As shown in [1], this protocol offers the optimal diversity/multiplexing trade-off among all the AF half-duplex protocols. The source $\mathrm{S}$, the destination $\mathrm{D}$, and the relay $\mathrm{R}$ are single-antenna terminals. $\mathrm{S}$ and $\mathrm{R}$ are assumed to be synchronized.

The channel impulse responses $\boldsymbol{h}_{i}$ are modeled as $L_{i} \times 1$ complex Gaussian random vectors with independent elements, i.e. $\boldsymbol{h}_{i} \sim \mathcal{C} \mathcal{N}\left(\mathbf{0}, \boldsymbol{C}_{i}\right)$, where $\boldsymbol{C}_{i}$ is diagonal and $i \in$ $\{\mathrm{SD}, \mathrm{SR}, \mathrm{RD}\}$. For the sake of the analysis, these channels are assumed (almost) time invariant for the duration of the two phases. Hence, the time index in the CFRs will be henceforth omitted. Moreover, they are assumed to be short enough to satisfy (3) above.

During the first phase, $\mathrm{S}$ trasmits the symbols $a_{1}(p, q)$ to $\mathrm{R}$ and $\mathrm{D}$. These are received as

$$
\begin{aligned}
y_{\mathrm{R}}(p, q) & =H_{\mathrm{SR}}(p) c_{1}(p, q)+\eta_{\mathrm{R}}(p, q), \\
y_{\mathrm{D}_{1}}(p, q) & =H_{\mathrm{SD}}(p) c_{1}(p, q)+\eta_{\mathrm{D}_{1}}(p, q),
\end{aligned}
$$

respectively. The noise terms are described as $\eta_{\mathrm{R}}(p, q) \sim$ $\mathcal{C N}\left(0, \sigma_{\mathrm{R}}^{2}\right)$ and $\eta_{\mathrm{D}_{1}}(p, q) \sim \mathcal{C N}\left(0, \sigma_{\mathrm{D}}^{2}\right)$. The signal $y_{\mathrm{R}}(p, q)$ is amplified by the relay as

$$
x_{\mathrm{R}}(p, q)=\lambda(p, q) y_{\mathrm{R}}(p, q),
$$

where the amplification factor $\lambda(p, q)$ is used to "regulate" the transmitted energy per FT point $(p, q)$ during the second phase. In the second phase, $\mathrm{S}$ and $\mathrm{R}$ send the symbols $a_{2}(p, q)$ and $x_{\mathrm{R}}(p, q)$ to $\mathrm{D}$, respectively. These are received as

$y_{\mathrm{D}_{2}}(p, q)=H_{\mathrm{SD}}(p) c_{2}(p, q)+H_{\mathrm{RD}}(p) c_{\mathrm{R}}(p, q)+\eta_{\mathrm{D}_{2}}(p, q),(8)$

where $\eta_{\mathrm{D}_{2}}(p, q) \sim \mathcal{C N}\left(0, \sigma_{\mathrm{D}}^{2}\right)$ and $c_{2}(p, q), c_{\mathrm{R}}(p, q)$ are as in (4) but with $a_{2}(p, q)$ and (complex) $x_{\mathrm{R}}(p, q)$ at the place of transmitted symbols. 


\section{Channel Estimation And EQUalization}

In order to better appreciate the needs of $\mathrm{D}$ for channel information, a simple, per-subcarrier, single-tap zero forcing (ZF) equalizer for recovering the transmitted data at the destination node is first considered here. It turns out that an additional interference term is present at $\mathrm{D}$, due to the use of OFDM/OQAM for forwarding at the relay. A simple way to cancel this term out is then described. Then, a least squares (LS) channel estimator is presented, based on the sparse preamble structure of [6]. This preamble, being the focus of this paper, will be optimized in the next section.

\section{A. ZF Equalization}

D needs to first estimate the virtual symbols $c_{k}(p, q)$ and from them detect the corresponding input symbols $a_{k}(p, q)$, $k=1,2$. The detected symbols are then OQAM-demodulated (real to complex (R2C) block in Fig. 1). Data recovery is performed at the end of the second phase. To see how this can be done, rewrite first eqs. (6) and (8):

$$
\begin{aligned}
y_{\mathrm{D}_{1}}(p, q)= & H_{\mathrm{SD}}(p) c_{1}(p, q)+\eta_{\mathrm{D}_{1}}(p, q) \\
y_{\mathrm{D}_{2}}(p, q)= & \lambda(p, q) H_{\mathrm{R}}(p) c_{1}(p, q)+H_{\mathrm{SD}}(p) c_{2}(p, q)+ \\
& I(p, q)+w_{2}(p, q),
\end{aligned}
$$

where $H_{\mathrm{R}}(p)=H_{\mathrm{SR}}(p) H_{\mathrm{RD}}(p)$ is the CFR of the S-R-D channel $^{2}$ of length $L_{\mathrm{R}}=L_{\mathrm{SR}}+L_{\mathrm{RD}}-1$,

$$
I(p, q)=j H_{\mathrm{RD}}(p) \sum_{(m, n) \in \Omega_{p, q}} \lambda(m, n) H_{\mathrm{SR}}(m) c_{1}(m, n)\langle g\rangle_{m, n}^{p, q}
$$

is an interference term induced by the relay forwarding operation, and

$$
\begin{gathered}
w_{2}(p, q)=H_{\mathrm{RD}}(p)\left[j \sum_{(m, n) \in \Omega_{p, q}} \lambda(m, n) \eta_{\mathrm{R}}(m, n)\langle g\rangle_{m, n}^{p, q}+\right. \\
\left.\lambda(p, q) \eta_{\mathrm{R}}(p, q)\right]+\eta_{\mathrm{D}_{2}}(p, q)
\end{gathered}
$$

is the composite noise at $\mathrm{D}$ in the second phase. Note that the time index $q$ refers here to the $q$ th OFDM/OQAM symbol of the data block transmitted during the corresponding phase.

$I(p, q)$ needs to be canceled out in (10) for a better detection performance. However, eq. (11) implies that D would also need an estimate of the S-R and R-D channels in order to cope with this interference term. This is more than commonly required from the destination node in the channel estimation literature for such systems (cf. e.g., [10]), namely estimates of the overall channels in the two paths from $\mathrm{S}$ to $\mathrm{D}$ only. One can see, however, that $I(p, q)$ can be approximated by using the assumption (underlying (3)) that $H_{\mathrm{SR}}(m) \approx H_{\mathrm{SR}}(p)$, for $(m, n) \in \Omega_{p, q}$. This way, $H_{\mathrm{SR}}(p)$ is factored out of the summation in (11) and the known channel $H_{\mathrm{R}}(p)$ appears. Moreover, $c_{1}(m, n)$ can be estimated from (6) based on the $H_{\mathrm{SD}}$ estimate, while the quantities $\langle g\rangle_{m, n}^{p, q}$ can be a priori known from the adopted $g$ [8]. Once $I(p, q)$ has been canceled out, and an estimate of $c_{1}(p, q)$ is available, a $\mathrm{ZF}$ equalizer can be applied in (10) too, to estimate $c_{2}(p, q)$.

\footnotetext{
${ }^{2}$ It is tacitly assumed here that the composite channel is short enough to meet the assumptions stated in Sec. II-A that validate (3).
}

\section{B. Channel Estimation}

$\mathrm{S}$ is assumed to employ a 3-symbol sparse preamble in the beginning of each phase, to assist the estimation of the channels at D. The usual sparse preamble structure is adopted, namely the first and third OFDM/OQAM symbols are set to zero, serving as guards against interference from data. The second (middle) OFDM/OQAM symbol has nonzero pilots at some positions described by the index set $\mathcal{P}=\left\{p_{1}, p_{2}, \ldots, p_{L}\right\}$ and zeros everywhere else. $L$, the number of pilot symbols, is assumed to be the minimum possible one, namely $L=\max \left(L_{\mathrm{SD}}, L_{\mathrm{R}}\right)$, and, of course, $L<<M$.

In the first phase, the training signals received at $\mathrm{R}$ and $\mathrm{D}$ are, respectively,

$$
\begin{aligned}
y_{\mathrm{D}_{1}}(p, 1) & =H_{\mathrm{SD}}(p) a_{1}(p, 1)+\eta_{\mathrm{D}_{1}}(p, 1), \\
y_{\mathrm{R}}(p, 1) & =H_{\mathrm{SR}}(p) a_{1}(p, 1)+\eta_{\mathrm{R}}(p, 1)
\end{aligned}
$$

with $p \in \mathcal{P}$. Note the absence of interference, due to the preamble sparsity. The AF operation at the relay during training is here defined as follows. The relay feeds its SFB with the amplified signals (7) at the pilot subcarriers, whereas it loads the remaining subcarriers with nulls. Moreover, the OFDM/OQAM symbols at $q=0,2$ are set to all zeros. This "recovers" the original preamble structure as sent by the source, yet with complex-valued inputs at the pilot subcarriers. Thus, the received signal at the destination in the second phase and at the pilot subcarriers can be expressed as

$y_{\mathrm{D}_{2}}(p, 1)=H_{\mathrm{SD}}(p) a_{2}(p, 1)+\lambda(p, 1) H_{\mathrm{R}}(p) a_{1}(p, 1)+w_{2}(p, 1)$

where now $w_{2}(p, 1)=H_{\mathrm{RD}}(p) \lambda(p, 1) \eta_{\mathrm{R}}(p, 1)+\eta_{\mathrm{D}_{2}}(p, 1)$ (cf. (12)). The latter has zero mean and variance $\sigma_{w_{2}}^{2}(p)=|\lambda(p, 1)|^{2} \theta_{\mathrm{RD}}^{2}(p) \sigma_{\mathrm{R}}^{2}+\sigma_{\mathrm{D}}^{2}$, where $\theta_{\mathrm{RD}}^{2}(p)$ equals $\mathcal{E}\left\{\left|H_{\mathrm{RD}}(p)\right|^{2}\right\}=\operatorname{Tr}\left(\boldsymbol{C}_{\mathrm{RD}}\right)=\theta_{\mathrm{RD}}^{2}$ and is therefore independent of $p$ (for uncorrelated channels as assumed here).

It will be convenient to write eqs. (13) and (15) in the following compact form:

$$
\begin{aligned}
{\left[\begin{array}{l}
\boldsymbol{y}_{\mathrm{D}_{1}} \\
\boldsymbol{y}_{\mathrm{D}_{2}}
\end{array}\right]=} & \sqrt{M}\left[\begin{array}{cc}
\boldsymbol{A}_{1} & \mathbf{0} \\
\boldsymbol{A}_{2} & \boldsymbol{\Lambda} \boldsymbol{A}_{1}
\end{array}\right]\left[\begin{array}{cc}
\boldsymbol{F}_{L \times L} & \mathbf{0} \\
\mathbf{0} & \boldsymbol{F}_{L \times L}
\end{array}\right] \times \\
& {\left[\begin{array}{c}
\boldsymbol{h}_{\mathrm{SD}} \\
\boldsymbol{h}_{\mathrm{R}}
\end{array}\right]+\left[\begin{array}{c}
\boldsymbol{\eta}_{\mathrm{D}_{1}} \\
\boldsymbol{w}_{2}
\end{array}\right] }
\end{aligned}
$$

where $\boldsymbol{y}_{\mathrm{D}_{k}}=\left[\begin{array}{llll}y_{\mathrm{D}_{k}}\left(p_{1}, 1\right) & y_{\mathrm{D}_{i}}\left(p_{2}, 1\right) & \cdots & y_{\mathrm{D}_{k}}\left(p_{L}, 1\right)\end{array}\right]^{T}$ $k=1,2$ and similarly for $\boldsymbol{\eta}_{D_{1}}$ and $\boldsymbol{w}_{2}$. Additionally, $\boldsymbol{A}_{k}=$ $\left.\operatorname{diag}\left(a_{k}\left(p_{1}, 1\right), a_{k}\left(p_{2}, 1\right)\right), \ldots, a_{k}\left(p_{L}, 1\right)\right)$, for $k=1,2, \boldsymbol{\Lambda}=$ $\operatorname{diag}\left(\lambda\left(p_{1}, 1\right), \lambda\left(p_{2}, 1\right), \ldots, \lambda\left(p_{L}, 1\right)\right)$, and the $L \times L$ matrix $\boldsymbol{F}_{L \times L}$ results from the $M$ th-order normalized DFT matrix by keeping its first $L$ columns and its $L$ rows indexed by $\mathcal{P}$. Here, it is assumed for simplicity and without loss of generality that $L_{\mathrm{SD}}=L_{\mathrm{R}}=L$ and that $M / L$ is an integer. If necessary, these can be satisfied by appending an appropriate number of zeros to the shorter impulse response. With a straightforward matching of terms, the above can be written as

$$
\begin{aligned}
\boldsymbol{y} & =\sqrt{M} \boldsymbol{X} \overline{\boldsymbol{F}}_{L} \boldsymbol{h}+\boldsymbol{w} \\
& =\boldsymbol{B} \boldsymbol{h}+\boldsymbol{w},
\end{aligned}
$$

where the matrix $\boldsymbol{B}=\sqrt{M} \boldsymbol{X} \overline{\boldsymbol{F}}_{L}$ is square of order $2 L$ and obviously nonsingular. The noise term $\boldsymbol{w}$ is a zero mean random vector with covariance matrix $\boldsymbol{C}_{\boldsymbol{w}}=\operatorname{diag}\left(\sigma_{\mathrm{D}}^{2} \boldsymbol{I}_{L}, \boldsymbol{C}_{\boldsymbol{w}_{2}}\right)$, 
where $\boldsymbol{C}_{\boldsymbol{w}_{2}}=\operatorname{diag}\left(\sigma_{w_{2}}^{2}\left(p_{1}\right), \sigma_{w_{2}}^{2}\left(p_{2}\right), \ldots, \sigma_{w_{2}}^{2}\left(p_{L}\right)\right)$. From eq. (17), the LS estimate of $\boldsymbol{h}$ and its covariance matrix result as

$$
\hat{\boldsymbol{h}}=\boldsymbol{B}^{-1} \boldsymbol{y}, \quad \boldsymbol{C}_{\hat{\boldsymbol{h}}}=\boldsymbol{B}^{-1} \boldsymbol{C}_{\boldsymbol{w}} \boldsymbol{B}^{-H} .
$$

The per-subcarrier amplification factor during the preamble period will be computed as (see also [10])

$$
\lambda(p, 1)=\sqrt{\frac{E_{\mathrm{R}}(p, 1)}{\theta_{\mathrm{SR}}^{2} a_{1}^{2}(p, 1)+\sigma_{\mathrm{R}}^{2}}},
$$

with $p \in \mathcal{P}$, where $E_{\mathrm{R}}(p, 1)$ is the energy assigned by the relay when forwarding the $p$ th pilot signal and $\theta_{\mathrm{SR}}^{2}$ is defined in a manner analogous to $\theta_{\mathrm{RD}}^{2}$ (and is hence independent of p).

\section{Optimal Training Design}

The aim here is to appropriately choose the pilot symbols $a_{k}(p, 1)$ and their positions $p \in \mathcal{P}$, so that the $\operatorname{MSE} \frac{1}{2 L} \operatorname{Tr}\left(\boldsymbol{C}_{\hat{\boldsymbol{h}}}\right)$ is minimized subject to a constraint on the total energy spent for transmitting (and forwarding) the preambles in the two phases. In a manner analogous to that followed in [10], one can show that the MSE here only depends on the energies of the pilot symbols and not on their specific values. This fact will be soon become apparent (cf. (21)).

\section{A. Problem Formulation}

Defining $\alpha_{k}(l)=a_{k}^{2}\left(p_{l}, 1\right)$ for $k=1,2$ and $e(l)=$ $E_{\mathrm{R}}\left(p_{l}, 1\right)$, one can state the preamble optimization problem as follows

$$
\min _{\boldsymbol{\alpha}_{1}, \boldsymbol{\alpha}_{2}, \boldsymbol{e}, E_{1}, E_{2}, \mathcal{P}} \frac{1}{2 L} \operatorname{Tr}\left(\boldsymbol{C}_{\hat{\boldsymbol{h}}}\right)
$$

such that (s.t.)

$E_{1}+E_{2}=E_{\mathrm{S}}, \sum_{l=1}^{L} \alpha_{1}(l)=E_{1}, \sum_{l=1}^{L} \alpha_{2}(l)=E_{2}, \sum_{l=1}^{L} e(l)=E_{\mathrm{R}}$, where $\boldsymbol{\alpha}_{k}$ and $e$ are $L \times 1$ vectors containing the $\alpha_{k}$ 's and $e$ 's, respectively, $E_{k}$ is the energy allocated to training in phase $k$ and $E_{\mathrm{R}}, E_{\mathrm{S}}$ are given energy budgets. The optimal placement of the pilot symbols $\mathcal{P}$ is also to be optimized.

A simplification of the above cost function will be quite helpful in the sequel. Using the formula for $\boldsymbol{C}_{\hat{\boldsymbol{h}}}$ from (18) and the definitions from (17), one can write $\operatorname{Tr}\left(\boldsymbol{C}_{\hat{\boldsymbol{h}}}\right)=$ $\frac{1}{M} \operatorname{Tr}\left\{\left(\boldsymbol{X}^{H} \boldsymbol{C}_{\boldsymbol{w}} \boldsymbol{X}\right)^{-1}\left(\overline{\boldsymbol{F}}_{L} \overline{\boldsymbol{F}}_{L}^{H}\right)^{-1}\right\}$, where the well known property of the trace operator for matrix products has been employed. Applying next the matrix inversion lemma in the $2 \times 2$ block diagonal (with diagonal blocks) matrix $\left(\boldsymbol{X}^{H} \boldsymbol{C}_{\boldsymbol{w}} \boldsymbol{X}\right)^{-1}$ results in $\operatorname{Tr}\left(\boldsymbol{C}_{\hat{\boldsymbol{h}}}\right)=\frac{1}{M} \operatorname{Tr}\left\{\boldsymbol{U}\left(\boldsymbol{F}_{L \times L} \boldsymbol{F}_{L \times L}^{H}\right)^{-1}\right\}$, where $\boldsymbol{U}$ is a diagonal matrix with its $l$-th diagonal element, $l=1,2, \ldots, L$, given by

$$
\begin{aligned}
u_{l}= & \frac{\sigma_{\mathrm{D}}^{2}+\sigma_{\mathrm{R}}^{2} \theta_{\mathrm{RD}}^{2}}{\alpha_{1}(l)}+\frac{\sigma_{\mathrm{D}}^{2} \theta_{\mathrm{SR}}^{2}}{e(l)}+\frac{\sigma_{\mathrm{D}}^{2} \sigma_{\mathrm{R}}^{2}}{\alpha_{1}(l) e(l)}+ \\
& \frac{\sigma_{\mathrm{D}}^{2} \theta_{\mathrm{SR}}^{2} \alpha_{2}(l)}{\alpha_{1}(l) e(l)}+\frac{\sigma_{\mathrm{D}}^{2} \sigma_{\mathrm{R}}^{2} \alpha_{2}(l)}{\alpha_{1}^{2}(l) e(l)} .
\end{aligned}
$$

Finally, by defining $v_{l}=\left[\left(\boldsymbol{F}_{L \times L} \boldsymbol{F}_{L \times L}^{H}\right)^{-1}\right]_{l, l}, l=1,2, \ldots, L$, the minimization can be based on the following equivalent expression for the cost function

$$
\operatorname{MSE}=\frac{1}{2 L} \operatorname{Tr}\left(\boldsymbol{C}_{\hat{\boldsymbol{h}}}\right)=\frac{1}{2 L M} \sum_{l=1}^{L} u_{l} v_{l} .
$$

\section{B. Optimal Energy Allocation Between Phases}

First, the optimal spliting of the total energy at the source in the two phases is investigated. Writing $\alpha_{k}(l)$ as a fraction of $E_{k}, \alpha_{k}(l)=f_{k}(l) E_{k}$, where $\sum_{l} f_{k}(l)=1$ for $k=1,2$, and setting $E_{2}=E_{\mathrm{S}}-E_{1}$, the MSE in (22) can be expressed as a function of $E_{1}$ only and the minimization is performed for $0 \leq$ $E_{1} \leq E_{\mathrm{S}}$. It can be proved that this function is monotonically decreasing and its minimum value is for $E_{1}=E_{\mathrm{S}}$, implying that $E_{2}=0$ and $\boldsymbol{\alpha}_{2}=\mathbf{0}$.

\section{Optimal Pilot Placement}

After incorporating the results of the previous subsections, the minimization problem is transformed into

$$
\begin{aligned}
& \min _{\boldsymbol{\alpha}_{1}, \boldsymbol{e}, \mathcal{P}} \frac{1}{2 L M} \sum_{l=1}^{L}\left[\frac{\sigma_{\mathrm{D}}^{2}+\sigma_{\mathrm{R}}^{2} \theta_{\mathrm{RD}}^{2}}{\alpha_{1}(l)}+\frac{\sigma_{\mathrm{D}}^{2} \theta_{\mathrm{SR}}^{2}}{e(l)}+\frac{\sigma_{\mathrm{D}}^{2} \sigma_{\mathrm{R}}^{2}}{\alpha_{1}(l) e(l)}\right] v_{l} \\
& \text { s.t. } \quad \sum_{l=1}^{L} e(l)=E_{\mathrm{R}}, \quad \sum_{l=1}^{L} \alpha_{1}(l)=E_{\mathrm{S}} .
\end{aligned}
$$

The optimization with $\stackrel{l=1}{l=1}$ ect to $\mathcal{P}$ can benefit from the

$$
\begin{aligned}
& \text { following lower bound [9] } \\
& \qquad \operatorname{Tr}\left(\boldsymbol{C}_{\hat{\boldsymbol{h}}}\right)=\frac{1}{M} \operatorname{Tr}\left\{\boldsymbol{U}\left(\boldsymbol{F}_{L \times L} \boldsymbol{F}_{L \times L}^{H}\right)^{-1}\right\} \geq \frac{1}{L} \sum_{l=1}^{L} u_{l} .
\end{aligned}
$$

Equality holds in (24) when the positioning set $\mathcal{P}$ is constructed by equispaced pilot positions (e.g. $p_{l}=\frac{M}{L}(l-1)$, for $l=1,2, \ldots, L)$.

\section{Optimal Allocation of Energy to Pilots}

By considering equispaced pilot positions, the minimization problem reduces to

$$
\begin{aligned}
& \min _{\boldsymbol{\alpha}_{1}, \boldsymbol{e}} \frac{1}{2 L^{2}} \sum_{l=0}^{L-1}\left[\frac{\sigma_{\mathrm{D}}^{2}+\sigma_{\mathrm{R}}^{2} \theta_{\mathrm{RD}}^{2}}{\alpha_{1}(l)}+\frac{\sigma_{\mathrm{D}}^{2} \theta_{\mathrm{SR}}^{2}}{e(l)}+\frac{\sigma_{\mathrm{D}}^{2} \sigma_{\mathrm{R}}^{2}}{\alpha_{1}(l) e(l)}\right] \\
& \text { s.t. } \sum_{l=1}^{L} \alpha_{1}(l)=E_{\mathrm{S}}, \quad \sum_{l=1}^{L} e(l)=E_{\mathrm{R}} .
\end{aligned}
$$

This can be readily solved using Lagrange multipliers. The conclusion is that the energy allocation should be uniform across the pilot symbols. This leads to $\alpha_{1}(l)=E_{\mathrm{S}} / L$ and $e(l)=E_{\mathrm{R}} / L$ for $l=1,2, \ldots, L$.

Remark. The above results are in line with those derived in [10] for the CP-OFDM-based system. This is not unexpected in view of the fact that the type of the preamble considered (i.e., sparse) removes intrinsic interference and brings the problem quite closely to that for CP-OFDM. Analogous results, for single-link (not relaying) OFDM/OQAM systems, were also shown in [6].

\section{Simulation Results}

In this section, some simulation results are reported, to corroborate the previous analysis. Filter banks with $M=256$, $K=3$ were used, employing the prototype filter designed in [2]. All channels were generated to undergo Rayleigh block fading with an exponential profile and lengths $L_{\mathrm{SD}}=32$, $L_{\mathrm{SR}}=16$ and $L_{\mathrm{RD}}=17$ (thus, in this case, $L=32$ ). The energy budgets were chosen as $E_{\mathrm{S}}=E_{\mathrm{R}}=L$, so as to have mean energy per pilot symbol equal to 1 . QPSK data were transmitted (with a unit energy per bit). Moreover, as usually assumed, $\sigma_{\mathrm{D}}^{2}=\sigma_{\mathrm{R}}^{2}$. The performance of the corresponding CPOFDM system is included, for the sake of the comparison, where a $\mathrm{CP}$ of minimum length (equal to the channel order) was assumed. 


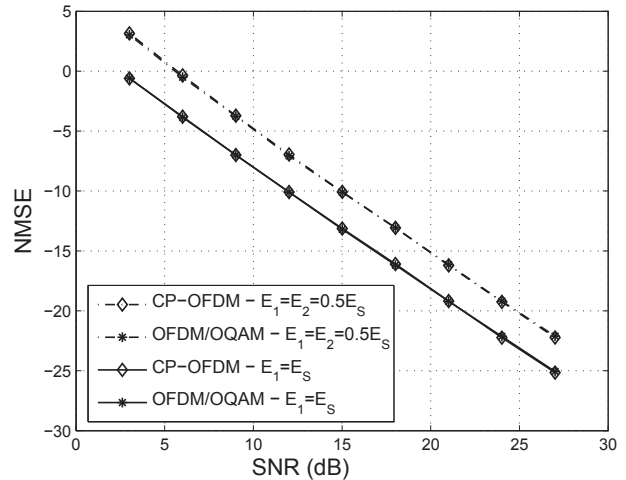

Fig. 2. NMSE performance of the optimal and suboptimal energy allocation between the two phases for $M=256, K=3$, and $L=32$.

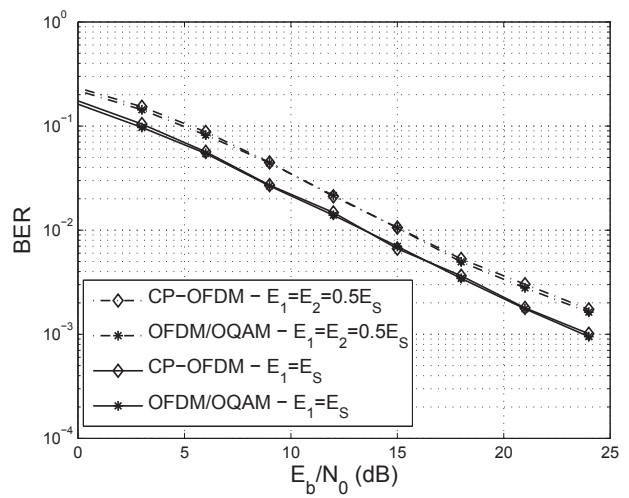

Fig. 3. BER for the data transmitted in the first phase $\left(a_{1}\right)$. Optimal and suboptimal energy allocation schemes are compared.

In Fig. 2, the normalized MSE (NMSE) $(\mathcal{E}(\| \boldsymbol{h}-$ $\left.\hat{\boldsymbol{h}}\left\|^{2} /\right\| \boldsymbol{h} \|^{2}\right)$ ) performance is depicted versus the signal to noise ratio (SNR), for both optimal $\left(E_{1}=E_{\mathrm{S}}\right)$ and suboptimal ( $\left.E_{1}=E_{2}=0.5 E_{\mathrm{S}}\right)$ source energy allocations between the two phases. All other training conditions hold as dictated by the optimal training design. As expected, the performance is significantly better when the optimal design is employed. Moreover, the two multicarrier systems perform similarly. Analogous results, omitted here due to lack of space, are obtained for the relay energy allocation.

In Figs. 3, 4, the (uncoded) bit error rate (BER) performances at the destination detector are shown for phases 1 and 2 , respectively. The SNR loss incurred by the $\mathrm{CP}$ redundancy in CP-OFDM was taken into account when calculating the corresponding BER. In the OFDM/OQAM-based relay, the amplification factors were chosen so as to have unit energy per information bit at the channel inputs of the S-R-D chain. One can observe a significant performance gain (of about 2$3 \mathrm{~dB}$ ) over the suboptimal source energy allocation. Moreover, and not unexpectedly, the two multicarrier systems perform similarly in the detection of the first phase data (cf. Fig. 3). In Fig. 4, the destructive effect of the interference term in (11) and the importance of its (approximate) cancellation are demonstrated. Observe the severe error floor in the optimal case without cancellation. On the other hand, no cancellation seems to be the best choice at low SNR values, because

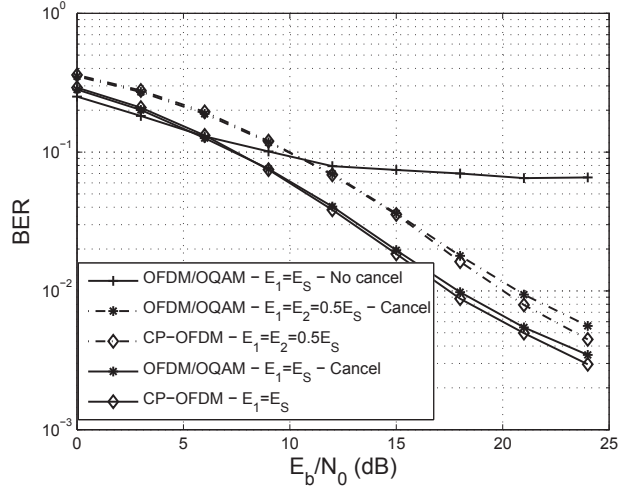

Fig. 4. As in Fig. 3, for the second phase transmission $\left(a_{2}\right)$.

of the errors incurred then at the interference approximation due to channel estimatior errors and $a_{1}$ decision error propagation. The somewhat worse than CP-OFDM performance of the cancellation-based equalizer at higher SNRs could be attributed to the augmentation of the composite noise at the OFDM/OQAM destination receiver because of the interference effect (cf. (12)). One should add to this the effect of the residual interference caused by the fact that (3) holds only approximately.

\section{ACKNOWLEDGMENT}

This work was supported by an FP7 grant, project EMPhAtiC (http://www.ict-emphatic.eu/).

\section{REFERENCES}

[1] K. Azarian, H. E. Gamal, and P. Schniter, "On the achievable diversitymultiplexing tradeoff in half-duplex cooperative channels," IEEE Trans. Info. Theory, Dec. 2005.

[2] M. G. Bellanger, "Specification and design of a prototype filter for filter bank based multicarrier transmission," in Proc. ICASSP'01.

[3] N. Benvenuto et al., "Analysis of channel noise in orthogonally multiplexed OQAM signals," in Proc. GLOBECOM'93.

[4] S. Berger et al., "Recent advances in amplify-and-forward two-hop relaying," IEEE Commun. Magazine, July 2009.

[5] B. Zafar, S. Gherekhloo, and M. Haardt, "Analysis of multihop relaying networks," IEEE Veh. Techn. Magazine, Sept. 2012.

[6] D. Katselis et al., "Preamble-based channel estimation for CP-OFDM and OFDM/OQAM systems: A comparative study," IEEE Trans. Signal Process., May 2010 (see arXiv:0910.3928v1 [cs.IT] for an extended version).

[7] D. Katselis et al., "On preamble-based channel estimation in OFDM/OQAM systems," in Proc. EUSIPCO'11.

[8] E. Kofidis et al., "Preamble-based channel estimation in OFDM/OQAM systems: A review," Signal Processing, July 2013.

[9] H. Lutkepohl, Handbook of Matrices, Wiley, 1996.

[10] C. Mavrokefalidis, A. Rontogiannis, and K. Berberidis, "Training design in single relay AF cooperative systems with correlated channels," in Proc. ICASSP' 11 .

[11] R. U. Nabar, H. Bölcskei, and F. W. Kneubühler,"Fading relay channels: Performance limits and space-time signal design," IEEE J. Sel. Areas in Commun., Aug. 2004.

[12] M. K. Ozdemir and H. Arslan, "Channel estimation for wireless OFDM systems," IEEE Commun. Surveys, vol. 9, no. 2, 2nd quarter 2007.

[13] PHYDYAS Project (ICT FP7). [Online] (http://www.ict-phydyas.org/).

[14] M. Shaat and F. Bader, "Comparison of OFDM and FBMC performance in multi-relay cognitive radio network," in Proc. ISWCS'12.

[15] P. Siohan, C. Siclet, and N. Lacaille, "Analysis and design of OFDM/OQAM systems based on filterbank theory," IEEE Trans. Signal Process., May 2002. 